\title{
In Situ Transmission Electron Microscopy
}

\section{Modulation of Transport in Graphene Nanoribbons}

\section{Supporting Information}

Julio A. Rodríguez-Manzo, ${ }^{\dagger}{ }^{+}$Zhengqing John Qi, ${ }^{\dagger,+}$ Alexander Crook, ${ }^{\dagger}$ Jae-Hyuk Ahn, ${ }^{\dagger}, \S$
A. T. Charlie Johnson, ${ }^{* \dagger}$ and Marija Drndić ${ }^{*}{ }^{\dagger}$

$\uparrow$ Department of Physics and Astronomy, University of Pennsylvania, Philadelphia, Pennsylvania 19104, United States

$\S$ Present address: Department of Electronic Engineering, Kwangwoon University, Seoul 01897, South Korea 


\section{SI-1: TEM graphene characterization.}

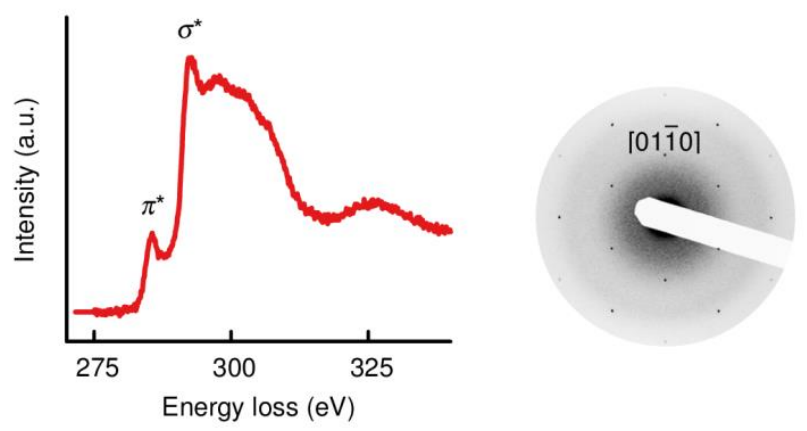

Figure SI-1: Energy electron-loss spectrum showing the carbon $K$-shell ionization edge (left) and electron diffraction pattern from a freestanding graphene strip fabricated in a TEM-compatible chip (right). Both signals were taken from an area $\sim 0.5 \mu \mathrm{m}^{2}$.

\section{SI-2: Graphene annealing via Joule heating.}

Graphene was annealed within the TEM column via Joule heating by applying a voltage $V_{b}$ between source and drain electrodes. The voltage was cycled in small increments up to $3 \mathrm{~V}$ to obtain current densities of the order of $10^{12} \mathrm{~A} \mathrm{~m}^{-2}$. Above these values the devices failed. Annealing caused a drop in GNR resistance and morphology changes in the GNRs associated with contamination removal and crystallization of amorphous carbon. 


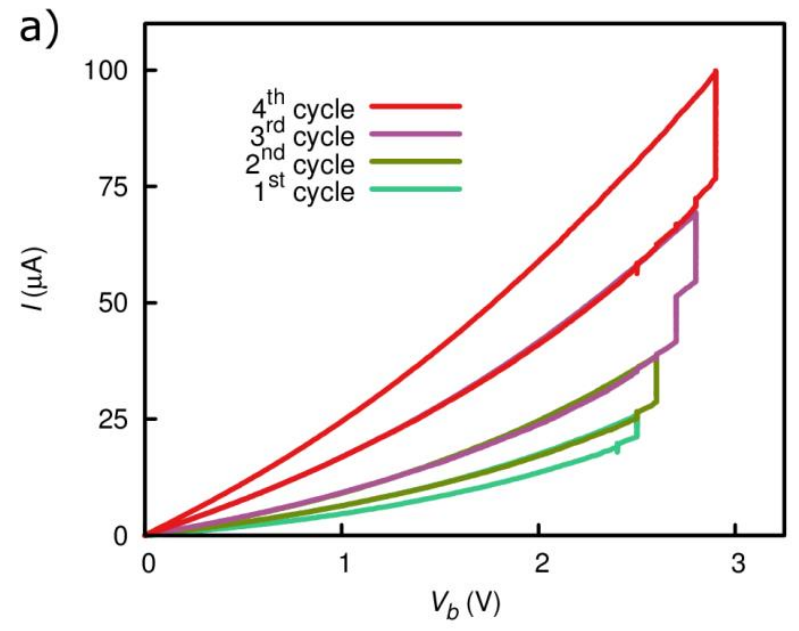

b)

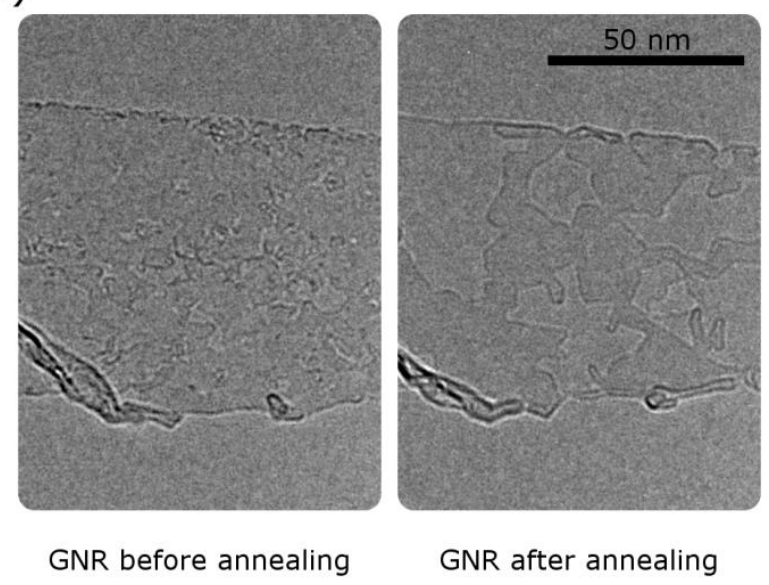

Figure SI-2: GNR annealing via Joule heating. a) Four annealing cycles in a 113-nm-wide GNR showing a decrease in GNR resistance as the $V_{b}$ is increased. b) TEM images of a GNR before and after annealing showing morphology changes.

\section{SI-3: Calculation details of the electrostatic potential and electric field.}

The electrostatic potential $V$ and electric field $\boldsymbol{E}=-\nabla V$ were numerically solved for vacuum $\left(\nabla^{2} V=0\right)$ between the three electrodes (source, drain and gate) considering the electrodes' surfaces as boundary conditions with equipotential values. The gate potential was set to $V_{g}$ while source and drain electrodes where grounded $(V=0)$. In the geometric model the electrodes' thickness is $50 \mathrm{~nm}$ except for the side-gate extension, carved from graphene in the experiment, whose thickness was set to $1 \mathrm{~nm}$. The $\operatorname{SiN}_{x}$ film was omitted from this analysis. Numerical solutions of the electrostatic equations were obtained with a physics modeling software (COMSOL Inc.). 


\section{SI-4: Transport measurements details and leakage current analysis.}

Graphene nanoribbons were connected to a voltage source (DAQ card National Instruments 6221 ) and a preamplifier to measure the current. A separate voltage source (multimeter Keithley 5417A) was used to supply the side-gate voltage. A custom LabVIEW program was used to set/sweep the voltages in addition to recording the electrical measurements. The leakage current $I_{\text {leakage }}$ of each device was established by measuring the current between gate and drain electrodes as a function of gate voltage $V_{g}$. In our measurements we capped $I_{\text {leakage }}$ to approximately \pm 30 nA by limiting the $V_{g}$ to a $\pm 10 \mathrm{~V}$ range, outside of which the low-stress 100 -nm-thick $\mathrm{SiN}_{x}$ film becomes more permissible to current. All quoted currents $I$ and, consequently, conductances $G$ in the main text were obtained after $I_{\text {leakage }}$ subtraction.

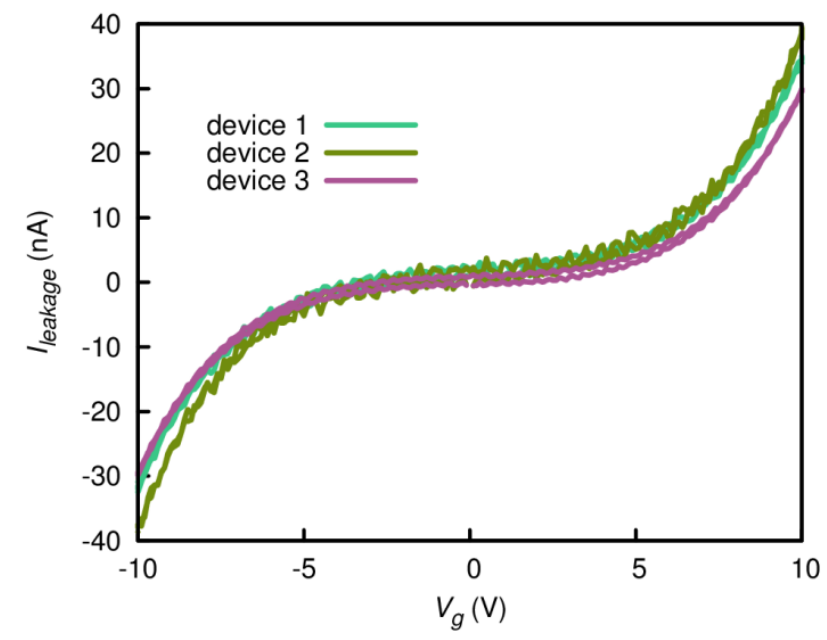

Figure SI-3: Leakage current between gate and drain electrodes as a function of gate voltage $\mathrm{Vg}$ from three independent devices. 